\title{
Wide Area RTK: A satellite navigation system based on precise real-time ionospheric modelling
}

\author{
J. M. Juan, ${ }^{1}$ J. Sanz, ${ }^{1}$ M. Hernández-Pajares, ${ }^{1}$ J. Samson, ${ }^{2}$ M. Tossaint, ${ }^{2}$ A. Aragón-Àngel, ${ }^{1}$ \\ and D. Salazar ${ }^{1}$ \\ Received 4 October 2011; revised 12 January 2012; accepted 1 March 2012; published 19 April 2012.
}

[1] The Wide Area Real Time Kinematic (WARTK) is an augmentation system concept for multi-frequency users based on precise real-time ionospheric modeling. It is able to provide a high accuracy and integrity GNSS positioning service over continental areas using the infrastructure of a network of permanent ground monitor stations, such as the European Geostationary Navigation Overlay Service (EGNOS) network of Ranging and Integrity Monitoring Stations (RIMS) in Europe. In this way, it allows an additional benefit to be obtained from these reference stations, that is, the network has the potential to support two independent systems: a satellite-based augmentation system, such as EGNOS, and a high-precision positioning service, based on WARTK. Indeed, thanks to the accuracy of the ionospheric corrections provided, WARTK users have available in real-time an extra constraint per satellite between the carrier phase ambiguities, which helps solve them quickly. Once such ambiguities have been solved, the GNSS user obtains navigation accurate to within $20 \mathrm{~cm}$ at the 95th percentile (about $10 \mathrm{~cm}$ RMS). Moreover, this precise positioning is achieved in a few minutes (with two frequency signals) or in a single epoch, after initial convergence of the tropospheric delay (with three frequency signals), even up to hundreds of kilometers away from the nearest reference station. While previous WARTK research has been devoted to implementing the concept and assessing its feasibility, considering in particular the accuracy achievable, the work reported in this paper focused on consolidating the results by analyzing a large and representative data set, and on deeper analysis of the integrity issue. It was carried out in the context of the Multi-constellation Regional System (MRS) project, within the European Space Agency GNSS Evolution Programme, with the aim of designing a high accuracy service for GPS and/or Galileo. Three months of actual data, from more than 25 permanent GPS stations in Europe, have been processed (some of them as a roving user), for high-, mid- and low-solar cycle conditions (in 2002, 2004 and 2006 respectively). In addition, several ionospheric storms occurred during the selected periods, with $D_{s t}$ values reaching up to $-150 \mathrm{nT}$. Results based on these data show that user domain integrity was maintained for baselines of up to $400 \mathrm{~km}$. At the 95 th percentile, the daily horizontal and vertical position errors were 20 and $30 \mathrm{~cm}$, respectively, and the corresponding protection levels were about 1 and $2 \mathrm{~m}$. The convergence time was around 5 minutes with actual GPS constellation data. The benefits of using a multi-constellation system were also studied, with simulated GPS and three-frequency Galileo data, showing that it is possible to reduce the convergence time to a few seconds.

Citation: Juan, J. M., J. Sanz, M. Hernández-Pajares, J. Samson, M. Tossaint, A. Aragón-Àngel, and D. Salazar (2012), Wide Area RTK: A satellite navigation system based on precise real-time ionospheric modelling, Radio Sci., 47, RS2016, doi:10.1029/2011RS004880.

\footnotetext{
${ }^{1}$ Research Group of Astronomy and Geomatics, Technical University of Catalonia, Barcelona, Spain.

${ }^{2}$ European Space Research and Technology Centre, European Space Agency, Noordwijk, Netherlands.

Copyright 2012 by the American Geophysical Union. 0048-6604/12/2011RS004880
}

\section{Introduction}

[2] Currently, two main types of high accuracy positioning techniques are typically used: precise point positioning (PPP) and real time kinematics (RTK). Both are based in the use of carrier phase measurements, which are more precise than code measurements but contain unknown ambiguities.

[3] In the PPP technique [see, e.g., Misra and Enge, 2010], a central processing facility (CPF) computes real- 
time precise orbits and clocks, which are provided to worldwide users for high accuracy navigation. In this way, once the navigation filter converges (which takes the best part of an hour), the user can achieve decimeter level accuracy (about 20 centimeters in terms of the positioning at the 95th percentile, hereinafter the statistic used to describe errors).

[4] RTK, on the other hand, is based on differential positioning, which relies on the hypothesis that for the user and a close reference receiver, with well known coordinates, the delays in the signal are similar and therefore common errors are canceled out [see, e.g., Misra and Enge, 2010]. Indeed, the user assumes that their measurements have the same ionospheric delays as the reference receiver. This assumption provides an additional relationship which makes it possible to obtain the user positioning to centimeter-level accuracy with a convergence time faster than PPP (i.e., a few minutes with two frequency signals). The problem with this technique is that this assumption for the ionosphere is typically only valid for baselines up to $10-15 \mathrm{~km}$.

[5] The Wide Area Real Time Kinematic (WARTK) technique, introduced 10 years ago and developed by gAGE/ UPC under several European Space Agency (ESA) funded projects, solves this problem by introducing a precise realtime ionospheric model to provide accurate ionospheric corrections to users [see Hernández-Pajares et al., 1999; Colombo et al., 1999].

[6] Unlike the RTK, an ionospheric model is computed in the WARTK CPF, which precisely captures the linear and larger scale electron content variations in real-time. The model tomographically maps the ionospheric state as measured by a network of permanent GNSS receivers, each separated by up to several hundreds of kilometers, following the same approach as detailed in previous papers of our research group (such as Juan et al. [1997] and HernándezPajares et al. [1997]). Using this ionospheric model at CPF level, it is possible to estimate the actual ionospheric delays affecting each satellite-receiver measurement.

[7] Once the ionospheric delays are computed for all satellites in view of the reference receivers, these values are transmitted to users, who can interpolate them to estimate their own ionospheric delays. Applying such ionospheric corrections, the user can quickly estimate the ambiguities, and even fix them when the corrections are sufficiently reliable. In this regard, it should be pointed out that, the results presented in this paper are obtained by fixing only the ambiguity in the wide-lane carrier phase, but not that for the short-lane ambiguity (see section 4).

[8] In this way, the WARTK technique typically provides accuracies within 20 centimeters (at the 95th percentile) and very quickly. The first indications of the feasibility of this approach for obtaining high accuracy positioning with integrity were found in the context of previous research activities supported by ESA [Hernández-Pajares et al., 2008]. An extensive explanation of proposed WARTK technique is given by Hernández-Pajares et al. [2010].

[9] Recently, a more thorough assessment of WARTK performance has been carried out as part of the Multiconstellation Regional System (MRS) project. In this project, a large data set with actual GPS data has been analyzed for three one-month periods under different solar conditions and the results have been extended to a multi-constellation scenario, paying special attention to integrity, in order to confirm its feasibility. The main results are presented in this paper.

\section{Performance Assessment}

[10] The assessment has been conducted using a reference station network built from the International GNSS Service (IGS) receivers, reproducing the topology of EGNOS RIMS network (see Figure 1). Data from all stations marked in blue, yellow and red on Figure 1 (but not the green symbols, which are the roving users) are employed by the CPF to calculate the WARTK corrections. Specifically, the data sets collected by these reference stations are processed by the WARTK CPF, where both an accurate ionospheric voxel model and a geodetic model are run cooperatively in the same filter to generate the WARTK corrections, emulating real-time conditions (see more details in the Description of the CPF Data Processing section).

[11] To assess the WARTK performance at the user level, four rover receivers (lliv, creu, bell, and ebre in Catalonia, NE Spain) were navigated using the WARTK corrections taking tlse (in Toulouse, France) as a reference station. The baselines of these roving receivers to the reference station range from $127 \mathrm{~km}$ (lliv) to $315 \mathrm{~km}$ (ebre) (see Table 1). The rovers are shown in green in Figures 1 and 2 (Figure 2 is a close-up of Figure 1). It must be pointed out that, although these receivers are located at permanent stations, i.e., with fixed coordinates, the processing was carried out as if they were roving receivers, that is, in pure kinematic mode and carefully emulating real time conditions, and without exploiting the fact that they are static.

[12] The rover navigates using the measurements of a near reference station (in this assessment tlse in red in Figures 1 and 2) and the slant total electron content (STEC) corrections computed from the surrounding reference stations (yellow and red stations in Figure 1), which are interpolated independently for each satellite at the rover location by means of a second order Taylor expansion, i.e., a quadratic fit of the user pierce point using the reference receiver pierce points of each given GNSS satellite. In practice, the interpolation is performed over the vertical total electron content (VTEC). This quadratic fit of VTEC requires measurements for at least seven reference stations to be available: one zeroorder term, two first-order terms, three second-order ones, and one extra observation, in order to have some redundancy to estimate a realistic indicator for the quality of the fit to be transmitted to the user.

\subsection{Experiment Description}

\subsubsection{Measurements}

[13] The IGS reference station network was initially deployed for scientific applications, mainly for precise global geodesy, and was not conceived as a testbed for navigation purposes. Thence, no continuity of service is guaranteed and some problems with the data collection (lack of data sets, receiver resetting, etc.) were encountered during long data collection campaigns. Nevertheless, in spite of these types of problems (related to the more demanding requirements of availability and continuity of a navigation service), the quality of the measurements can be considered good enough. 


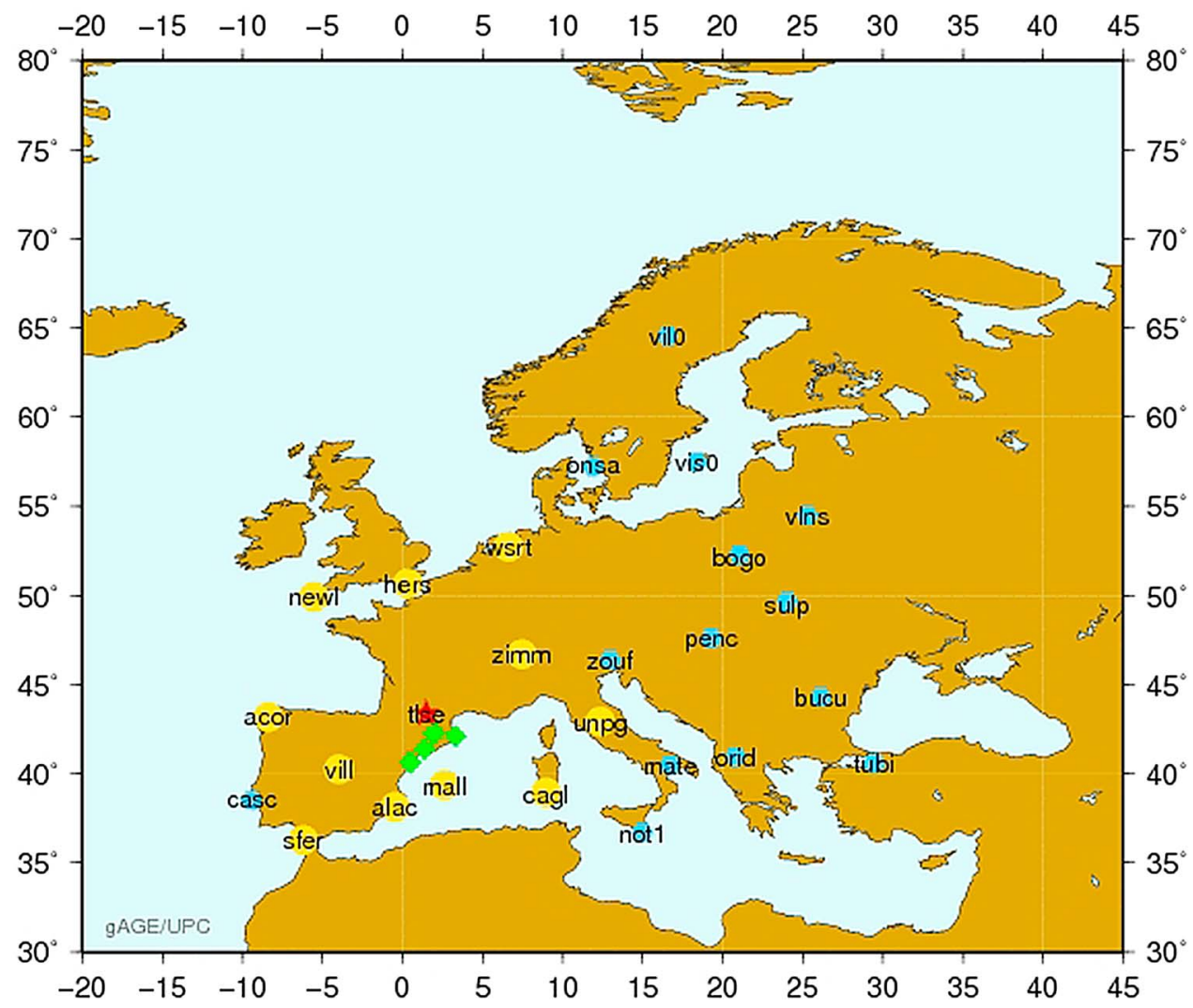

Figure 1. Geographical distribution of receivers in the data campaigns. The yellow and blue symbols correspond to reference station network used by the central processing facility to calculate the WARTK corrections. The yellow symbols indicate the stations whose ionospheric corrections are involved in the user receiver interpolation to estimate the ionospheric refraction at the rover location; the green ones indicate the rover receivers; and the red one represents the reference station for differential navigation.

[14] The data sets collected from the IGS receivers (i.e., reference stations and rovers) are dual frequency code and carrier phase measurements provided by Ashtech, AOAD, Trimble, JAVAD and Leica dual frequency receivers, among other commercial brands. The IGS receiver data files used in this study were stored with a sampling rate of 30 seconds. This relatively low sampling rate is not a limiting factor for the purposes of this assessment because continuity of service is not considered, and on the other hand, the user domain solutions obtained in less than 30 seconds are typically correlated due to the navigation filter used. Nevertheless, analysis at higher rates (such as $1 \mathrm{~Hz}$ ) can be expected to provide somewhat better performance due to the convergence of certain carrier phase ambiguities (such as the wide-lane), in such a way that the accuracy and integrity obtained in this study at 30 seconds may be considered a conservative assessment of the performance achievable.

\subsubsection{Ionospheric Conditions}

[15] WARTK users apply accurate correction estimates, calculated in real-time by the WARTK CPF, as additional data. As is well known, the state of the ionosphere depends on solar activity, which follows an 11-year cycle (solar cycle). In this context, the approximately three-month data set used for this study has been split into three different periods of four weeks, covering maximum, intermediate and minimum phases of the solar cycle. The first period analyzed corresponds to 3 May to 30 May in 2002,that is, close to the solar maximum (i.e., from day of year (DOY) 123 to 150 of 2002); the second to 28 July to 24 August 2004 (i.e., from DOY 210 to 237 of 2004) under mid-solar cycle conditions; and the third is from 19 November to 16 December 2006, with low solar activity (i.e., from DOY 323 to 350 of 2006). The radio solar flux from 2001 to 2006 is plotted in Figure 3, with the chosen periods marked in blue. It is worth noting that the periods used for the 2002, 2004 and 2006 data campaigns were selected in order to ensure that all the days in 2002 had solar flux of over $80 \%$ of the solar cycle values, those of 2004 data collection campaign over $50 \%$, and the days in the weeks of 2002 over $20 \%$.

[16] During the months studied ionospheric conditions varied, including highly disturbed periods, which are characterized by the Dst index [Datta-Barua et al., 2005] reaching values up to $-150 \mathrm{nT}$ (see Figure 4); this corresponds to

Table 1. WGS84 Coordinates of the Roving Receivers Used in This Assessment ${ }^{\mathrm{a}}$

\begin{tabular}{lcccc}
\hline Sites & Lon $(\mathrm{deg})$ & Lat $(\mathrm{deg})$ & $\mathrm{R}(\mathrm{km})$ & Baseline to TLSE $(\mathrm{km})$ \\
\hline lliv & 1,973 & 42,287 & 6369,897 & 127 \\
creu & 3,316 & 42,127 & 6368,622 & 204 \\
bell & 1,401 & 41,409 & 6369,608 & 218 \\
ebre & 0,492 & 40,631 & 6369,151 & 315 \\
\hline
\end{tabular}

${ }^{\mathrm{a}}$ The baselines to the reference station are also listed. 


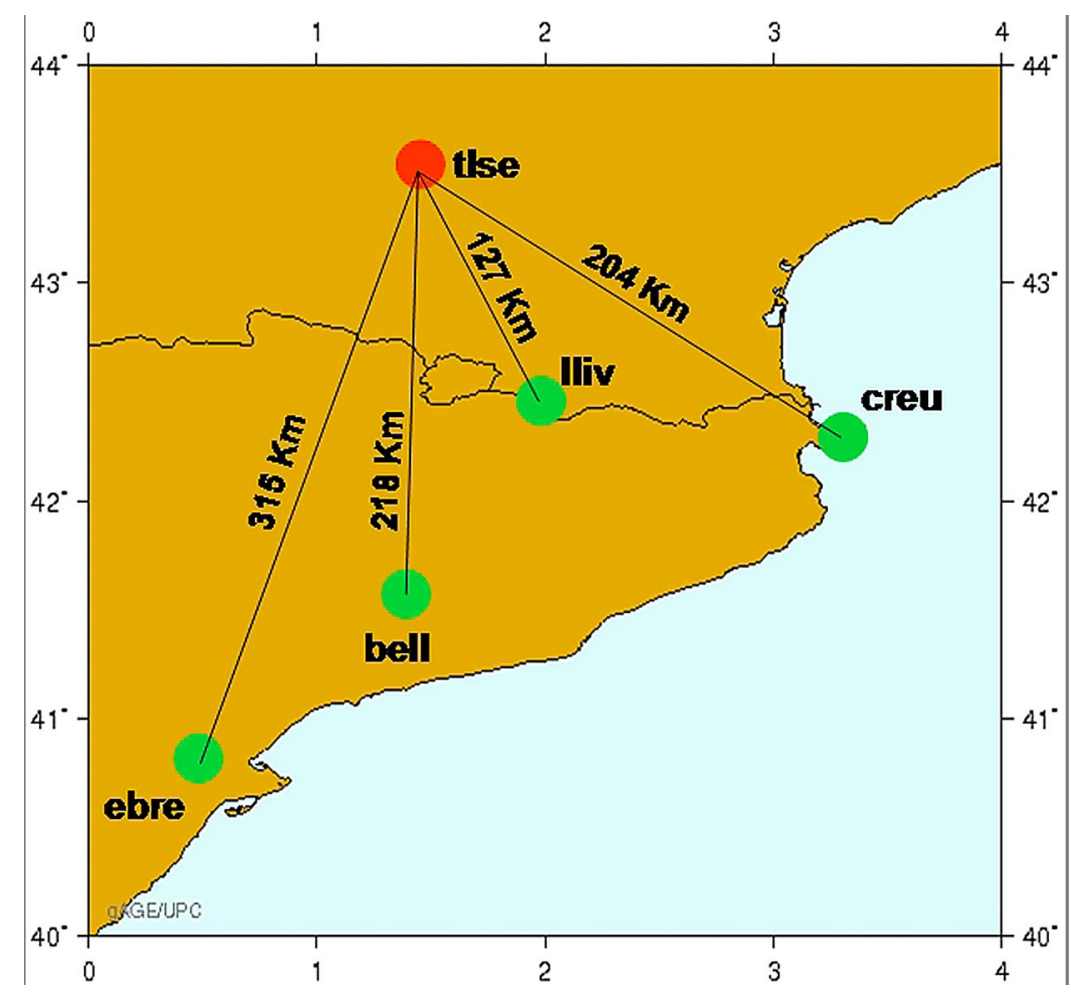

Figure 2. Zoom of Figure 1, showing the rover receivers in green (lliv, creu, bell, ebre) and the reference station (tlse) in red. The baselines between rovers and reference station are also drawn on the map.

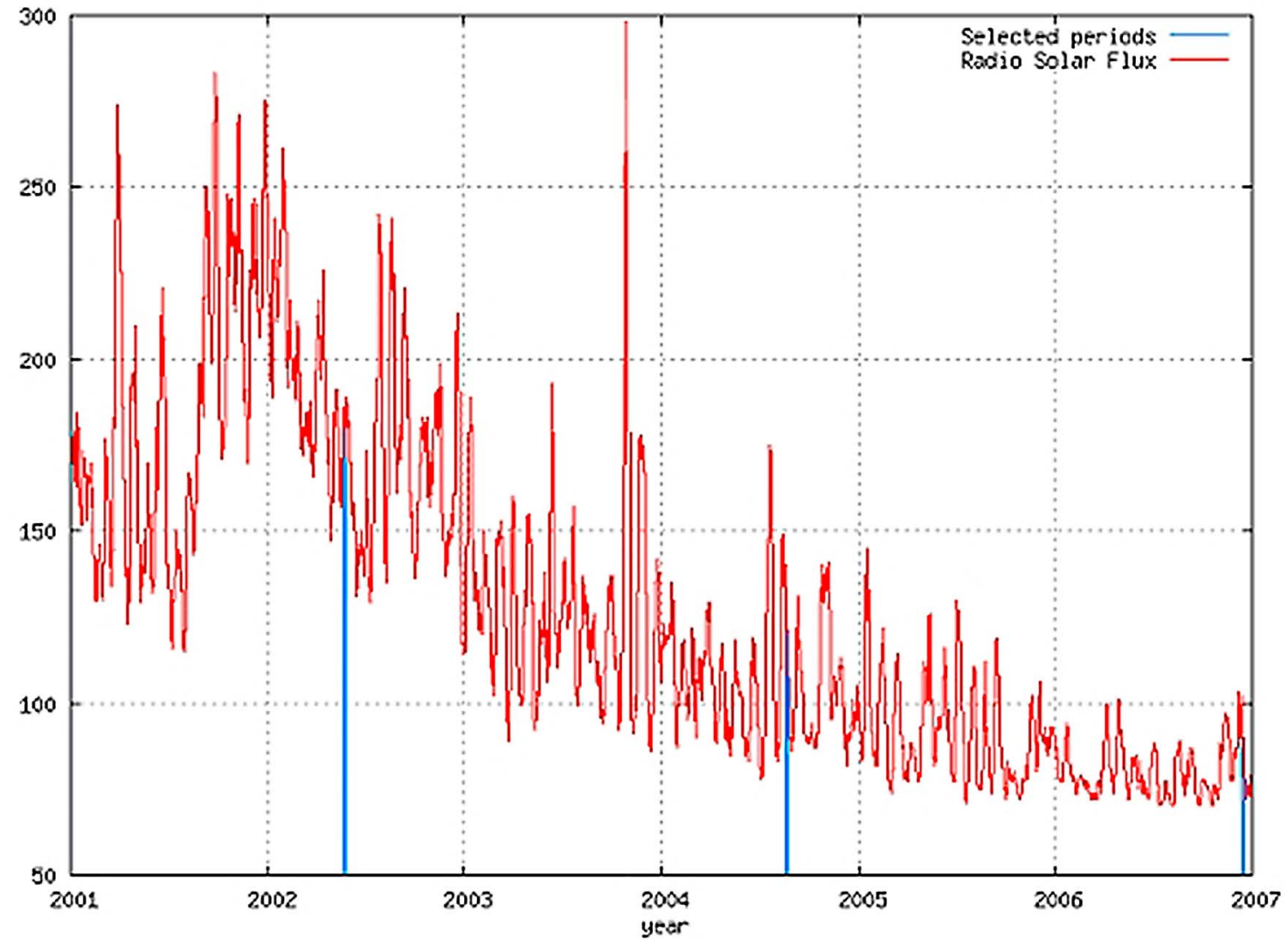

Figure 3. F10.7 Radio solar flux from the end of 2001 to 2007. The flux for the three studied periods is shown in blue. 

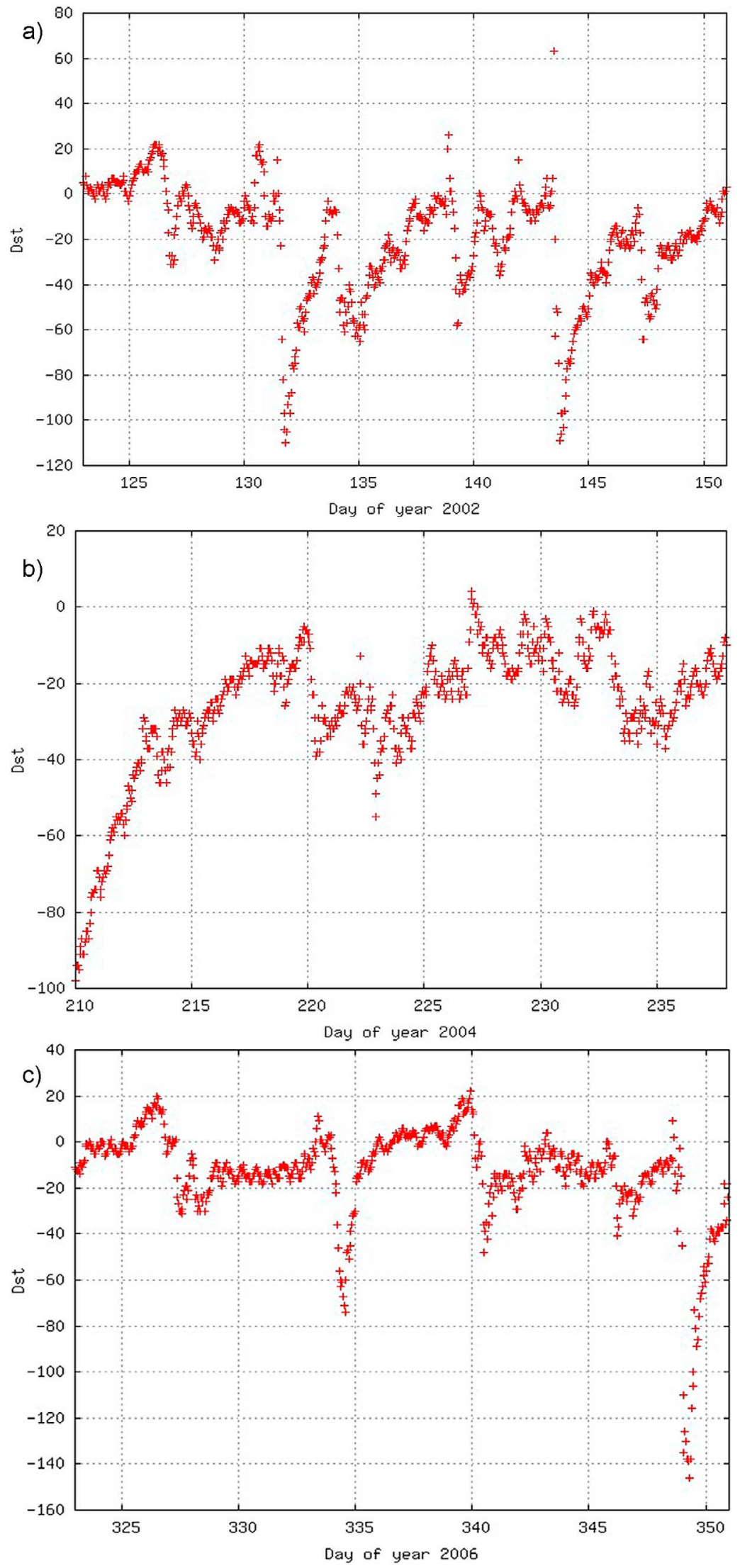

Figure 4. The 3-hour Dst index values for the studied days: (a) 2002 (from 3 May to 30 May, i.e., from DOY 123 to 150), (b) 2004 (from 28 July to 24 August, i.e., from DOY 210 to 237), and (c) 2006 (from 19 November to 16 December, i.e., from DOY 323 to 350 ). 
a cumulative distribution function of $99.7 \%$ since 1957 (i.e., it is in the top $0.3 \%$ of all the Dst values for the 50 years up to and including the study period). During such disturbed periods the quality of the ionospheric corrections could be degraded significantly [see, e.g., Orús et al., 2007]. Indeed, in some of these periods, the differential ionospheric delays between user and the nearest reference receiver reached values up of to $13 T E C U s$ (during more quiet periods, the typical values are below 7TECUs). Nevertheless, it must be taken into account that, on one hand, the ionospheric disturbances only affect the WARTK technique during filter convergence and, on the other hand, the main issues affecting the technique concern the non-linear differential ionospheric delays that may exist between the user and reference receivers. An important example of these phenomena for precise GNSS positioning are the medium scale traveling ionospheric disturbances (MSTIDs) [see, e.g., Wanninger, 2004; Hernández-Pajares et al., 2006].

\section{Description of the CPF Data Processing}

[17] As mentioned before, the data sets collected by the EGNOS-like reference station network are processed by the WARTK CPF, where both accurate ionospheric voxel and geodetic models are run using the same filter (emulating real-time conditions) to calculate the WARTK corrections (as in the work by Hernández-Pajares et al. [2002]). The measurements are taken at a 30 -second sampling rate, updating the Kalman filter every 5 minutes.

[18] The IGS precise predicted orbits (ultra-rapid products), which are provided up to 24 hours in advance, have been used for the satellite coordinates because they are openly available and provide sufficient accuracy (typically of better than $10 \mathrm{~cm}$, the error generally being negligible when navigating in differential mode). Such orbital information could also be predicted in post-processing mode, using any standard scientific software package with a precise dynamic model, fed with data from the previous day. In this work, however, we have focused on the more critical issue, i.e., the corrections which are to be calculated in real-time (satellite clocks and, especially, the ionospheric delays).

[19] The preprocessing of observables is carried out in a similar way by both CPF and users involving, in particular, the detection of carrier-phase cycle slips. After that, the filter estimation has three elements, which work together in a synergistic way: the geodetic sub-filter, the ionospheric subfilter and the iono-and-geo-free sub-filter. Below, we provide a brief explanation of the equations and parameters involved in the CPF filter (for a given satellite $j$ and receiver $i$ ):

[20] Geodetic sub-filter: From the ionosphere-free carrier phase $(L c)$ and pseudorange $(P c)$ combinations of dual frequency measurements, the satellite and receiver clock offsets $\left(\delta t^{j}\right.$ and $\left.\delta t_{i}\right)$, the carrier phase ambiguities $(B c)$ and the receiver zenith tropospheric delay $(T z)$ are estimated using the following expressions:

$$
\begin{aligned}
& \Delta P c_{i}^{j}=c\left(\delta t_{i}-\delta t^{j}\right)+M_{i}^{j} \cdot T z_{i} \\
& \Delta L c_{i}^{j}=c\left(\delta t_{i}-\delta t^{j}\right)+M_{i}^{j} \cdot T z_{i}+B c_{i}^{j}
\end{aligned}
$$

where $\Delta$ is the pre-fit residual of the observation (i.e., the measurement corrected by the known or nominal effects), $c$ is the speed of light in a vacuum, and $M_{i}^{j}$ is an obliquity factor (the tropospheric mapping).

[21] Iono-and-geo-free sub-filter: From the MelbourneWübbena combination $(\mathrm{Lw}-\mathrm{Pn})$ the ambiguity of the widelane combination of carrier phase $(B w)$ is given by:

$$
L w_{i}^{j}-P n_{i}^{j}=B w_{i}^{j}
$$

where $L w$ is the wide-lane combination of carrier phases, and $P n$ is the narrow-lane combination of code pseudoranges.

[22] Ionospheric sub-filter: From the geometry-free carrier phase and pseudorange combinations $\left(L_{I}, P_{I}\right)$, the mean electron density per voxel $\left(\mathrm{Ne}_{k}\right)$, corresponding to an optimal ionospheric tessellation, and the differential code biases (DCBs) for both satellites and receivers are estimated from the following equations:

$$
\begin{aligned}
P_{I_{i}^{j}}^{j} & =\alpha_{I} S T E C_{i}^{j}+D C B_{i}+D C B^{j} \\
L_{I_{i}^{j}}^{j} & =\alpha_{I} S T E C_{i}^{j}+D C B_{i}+D C B^{j}+\alpha_{w}\left(B w_{i}^{j}-B c_{i}^{j}\right)
\end{aligned}
$$

where:

$$
S T E C_{i}^{j}=\sum_{k} N e_{k} \cdot l_{k}
$$

is the STEC for a given ray between the station $i$ and satellite $j$ and the summation is extended to all voxels crossed, and $l_{k}$ is the length of the ray across the $k$ th-voxel. $\alpha_{I}=1.05 \times 10^{-17}$ is a conversion factor between $e^{-} / \mathrm{m}^{2}$ and meters of delay in $L_{I}=L 1-L 2$ units, and $\alpha_{w}=$ $\frac{f_{1}^{2}-f_{2}^{2}}{f_{1} f_{2}}=0.50$ (for the GPS frequencies $f_{1}$ and $f_{2}$ ) is a factor which is derived from the relationship between $L_{I}$, $L c$ and $L w$.

[23] Double-difference ambiguity fixing. The ambiguities $B c$ and $B w$ are estimated in the navigation filter as real numbers from previous equations, and thence, the associated ambiguities for the carrier signals $L 1$ and $L 2$ can be derived. Once such estimates converge, an additional constraint can be introduced to the ambiguities by assuming that they are integer multiples of the wavelength in double-differences (DDs) between receivers and satellites.

[24] Note that the non-differenced STEC for any satellitereceiver pair can also be calculated from the geometry-free combination of carriers, whose ambiguities have been previously fixed after removing the DCBs (see equation (3)).

[25] It must be stressed that one of the main outputs of this combined filter is the ionospheric corrections for the satellites in view from the reference station network, which consist of the precise non-differenced STEC values of every GNSS satellite in view of every ground receiver of the permanent network. This is actually the new information which is being broadcast to the user compared to the case of RTK or other precise GNSS positioning techniques based on GNSS carrier phase data, and it allows the RTK service to be extended from a few tens of kilometers to hundreds of kilometers away from the nearest reference station, quickly achieving a high accuracy. This correction broadcasting requires a very low bandwidth, less than 0.5 kbytes per five minutes (for instance, 7 coefficients $\times 20$ satellites by in view $\times 2$ bytes per coefficient $=280$ bytes per refresh), a short enough ionospheric updating time for most situations. 
[26] Although in a consolidated implementation of the system the CPF would run continuously, for this assessment an independent CPF run was performed each day in order to study the CPF convergence. An initial time period of 4 hours was considered for each daily run to ensure stable WARTK corrections. From this time on, the differential corrections provided by the CPF were assumed to be valid for the user to navigate.

\section{Description of the User Data Processing}

[27] The user navigates in differential mode with respect to the nearest reference station $(k)$ and a reference satellite $(m)$, running similar equations to those in the CPF but in DD mode:

[28] Geodetic sub-filter: The user estimates the coordinates, the zenith tropospheric delay and the double differences of carrier phase ambiguities using the following equations:

$$
\begin{aligned}
& \Delta P c_{i, k}^{j, m}=-\left(\hat{\rho}_{i}^{j}-\hat{\rho}_{i}^{m}\right) \cdot \Delta \vec{R}_{i}+\left(M_{i}^{j}-M_{i}^{m}\right) \cdot T z_{i} \\
& \Delta L c_{i, k}^{j, m}=-\left(\hat{\rho}_{i}^{j}-\hat{\rho}_{i}^{m}\right) \cdot \Delta \vec{R}_{i}+\left(M_{i}^{j}-M_{i}^{m}\right) \cdot T z_{i}+B c_{i, k}^{j, m}
\end{aligned}
$$

where $\Delta P c$ and $\Delta L c$ are the pre-fit residuals of code (Pc) and carrier $(\mathrm{Lc})$ ionosphere-free combinations of measurements (in DD mode), $\hat{\rho}$ is the line-of-sight unitary vector and $\Delta \vec{R}$ is the position correction with respect to a nominal value. In equation (5), it is assumed that the reference station measurements are corrected by the tropospheric delay, which is known at the CPF level.

[29] Iono-and-geo-free sub-filter: From the MelbourneWübbena combination $(L w-P n)$ the ambiguity of the widelane combination of carrier phase $\left(B w_{i, k}^{j, m}\right)$ is estimated as in the CPF, but in DD mode:

$$
L w_{i, k}^{j, m}-P n_{i, k}^{j, m}=B w_{i, k}^{j, m}
$$

This DD wide-lane ambiguity can be fixed by rounding the smoothed value to the nearest integer, typically in less than 5 minutes.

[30] Ionospheric sub-filter: Using the broadcast ionospheric corrections $\left(S T E C_{k}^{j}\right)$, the user interpolates the STEC at its location $\left(S T E C_{i}^{j}\right)$ and calculates the DD of STECs $\left(\right.$ STEC $\left._{i, k}^{j, m}\right)$ [Hernández-Pajares et al., 2004], which allows the following link between the double-difference ambiguities and carrier measurements in the geometry-free combination to be defined:

$$
L_{I, k}^{j, m}-\alpha_{I} S T E C_{i, k}^{j, m}=\alpha_{W}\left(B w_{i, k}^{j, m}-B c_{i, k}^{j, m}\right)
$$

Note that the code measurements are not needed in the user ionospheric sub-filter. It should also be pointed out that the user navigation filter processes all satellites in view, incorporating the ionospheric corrections for the available satellites as additional constraints weighted by the interpolation error.

[31] Combining equations (6) and (7), the user can obtain a good estimate of the ionosphere-free ambiguity $(B C)$, allowing rapid convergence of the precise positioning in the geodetic sub-filter, equation (5). In this sense, notice that the ionospheric sub-filter is basically used to speed-up the navigation filter solution, which only requires the wide-lane ambiguity to be fixed. For this purpose, ionospheric corrections with an accuracy better than $1 T E C U$ are sufficiently good (this error level is significantly better than the code pseudorange noise in the ionosphere free combination, equation (5), and this is the reason why the filter convergence is faster).

[32] Once the filter has converged, the position is mainly determined by the geodetic sub-filter, equation (5), which uses the ionosphere-free combination of measurements, and thence, it is not directly affected by the ionospheric disturbances.

[33] Finally, it should be underlined that, in addition to the coordinates, the user navigation filter also provides their covariance matrix, which can be projected as vertical and horizontal error components to calculated the corresponding standard deviations and the protection levels (see below).

\section{User Domain Performance}

[34] Regarding the number of satellites in view, the distributions for 2004 and 2006 were quite similar with typically eight, while slightly fewer satellites were in view (around seven) in 2002. This reduction in the number of tracked satellites, together with the higher solar activity conditions explain a decrease in the number of ionospheric corrections available in 2002. Furthermore, there were consistently far fewer wide-lane DDs available in 2002 (around four), which again can be attributed to the smaller number of satellites tracked, compared with 2004 and 2006. These factors, explain the corresponding worsening in the convergence time seen in Figure 5 in 2002, under solar maximum conditions.

\subsection{Convergence Time}

[35] Figure 5 shows a comparison of the accuracy achieved as a function of the time elapsed since user receiver reset when using -in red- (or when ignoring -in green-) the WARTK ionospheric corrections for each data collection campaign: 2002 (top), 2004 (middle), and 2006 (bottom). In all three cases, the results have been calculated over the same data sets (one month each year) and under the same conditions, except for the usage of ionospheric corrections. They are generated averaging the results for each epoch (time since last reset) of all resets and stations of each data collection campaign, for both the horizontal and vertical components. Given that these accuracy curves tend to decrease as a function of time (indicating improving accuracy), convergence times can be compared by monitoring the time taken to achieve certain levels of accuracy. Accordingly, each plot contains a table summarizing the time to reach the thresholds of $10 \mathrm{~cm}$ and $20 \mathrm{~cm}$ in horizontal and $15 \mathrm{~cm}$ and $30 \mathrm{~cm}$ in vertical error components.

[36] The results show a strong reduction in the convergence time, especially in the horizontal component, which is reduced by a factor of 5 (2002) to 8 (2004 and 2006), allowing a horizontal accuracy of $20 \mathrm{~cm}$ to be reached within 5 minutes, and of $10 \mathrm{~cm}$ within 10 minutes, in contrast to the better part of an hour needed in the case of the classical differential positioning. In the vertical component, the reduction is more moderate (typically, by a factor of 3 ), but $30 \mathrm{~cm}$ vertical accuracy is reached in less than 10 minutes for 2004 and 2006 data, and in 15 minutes for 2002. Table 2 

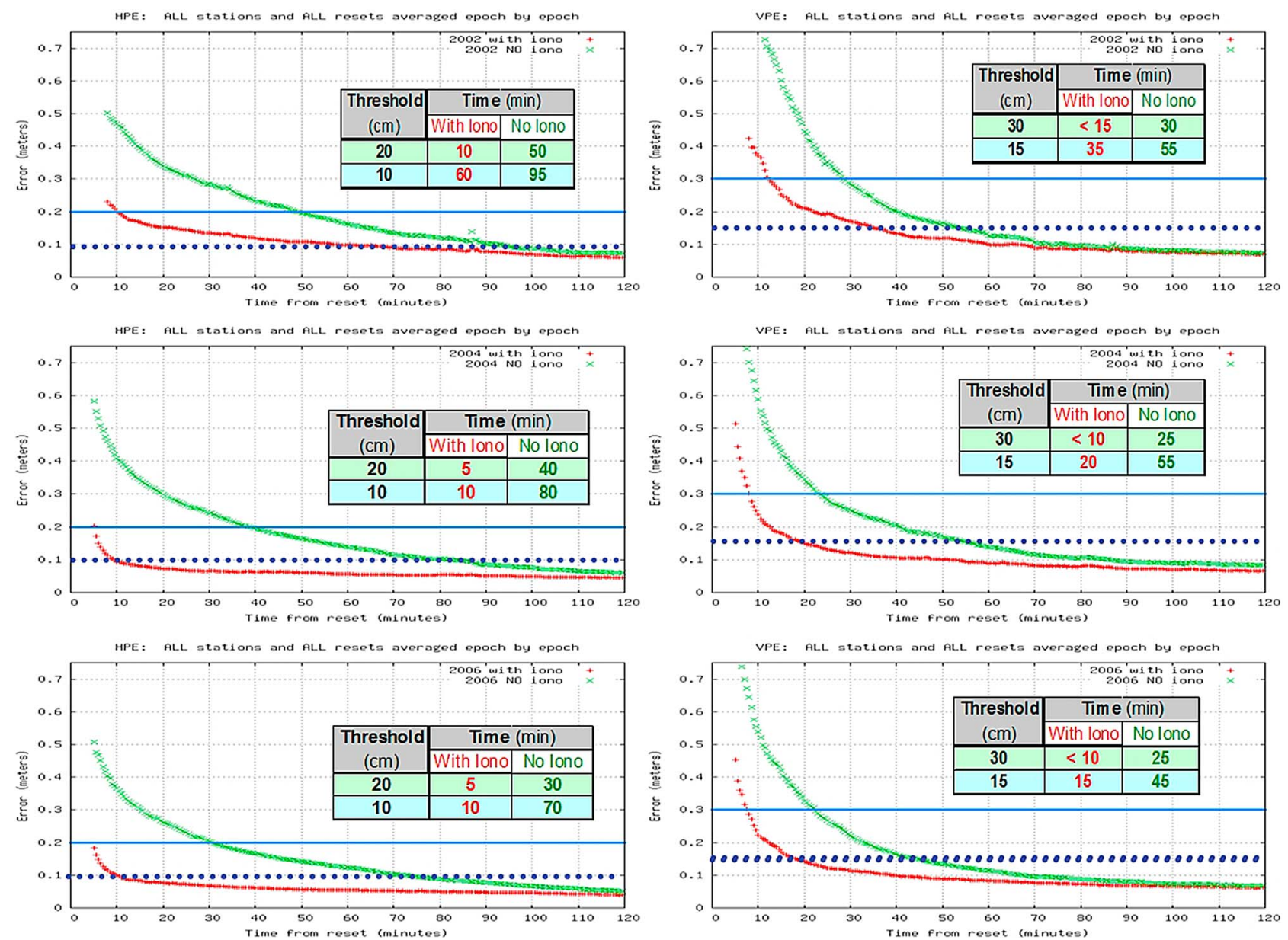

Figure 5. Convergence time curves: (left) horizontal and (right) vertical accuracy as a function of the time since user receiver reset. The red curve corresponds to the WARTK positioning (i.e., using ionospheric corrections to accelerate the filter convergence). The green curve corresponds to the standard differential positioning (i.e., without using ionospheric corrections). The data collection campaigns of (top) 2002, (middle) 2004, and (bottom) 2006. The reference accuracy thresholds for the convergence time (see Table 2) are also indicated as light blue continuous and dark blue dotted horizontal lines.

summarizes these results, providing a comparison of the typical convergence time to reach the $10 \mathrm{~cm}$ and $20 \mathrm{~cm}$ (in horizontal), and $15 \mathrm{~cm}$ and $30 \mathrm{~cm}$ (in vertical) error thresholds.

[37] Also note in Figure 5 that the convergence time curves using ionospheric corrections (in red) for the 2004 and 2006 data collection campaigns follow a similar pattern (in both error components, horizontal and vertical), reaching convergence in few minutes (after an initial rapid drop), while the curve for the 2002 data collection campaign has a more gradual slope and takes more time to stabilize. This worsening in the 2002 results is mainly related to the smaller number of satellites with ionospheric corrections tracked by the reference stations, as explained in the previous section. Nevertheless, even in this difficult scenario the convergence time is significantly reduced compared to the standard differential positioning, reaching horizontal and vertical of accuracies of $20 \mathrm{~cm}$ and $30 \mathrm{~cm}$ in about 10 minutes. These results suggest that in nominal conditions typical convergence times of 5 and 10 minutes can be expected for the horizontal and vertical error components (see Table 2).
[38] It should be pointed out that the periods analyzed contain several days with intense geomagnetic activity (see Figure 4). Although the real-time ionospheric model can be processed accurately by the permanent receivers during days of extreme geomagnetic activity [Hernández-Pajares et al., 2002], there could be difficulties fixing the two ambiguities for precise navigation [see Grejner-Brzezinska et al., 2006]. Nevertheless, our results were not significantly affected by geomagnetic activity as can be seen in Figure 4 (under values of Dst $=-60 \mathrm{nT}$ ), thanks to the fact that

Table 2. Summary of Convergence Times Obtained With Actual Dual-Frequency Single Constellation GPS Data, Up to the Stated, Arbitrarily Chosen, Horizontal and Vertical Accuracy Thresholds Arbitrarily Chosen

\begin{tabular}{lcc}
\hline Threshold & Convergence With Iono & Time No Iono \\
\hline H: $20 \mathrm{~cm}$ & $\sim 5 \mathrm{~min}$ & $\sim 40 \mathrm{~min}$ \\
H: $10 \mathrm{~cm}$ & $\sim 10 \mathrm{~min}$ & $\sim 80 \mathrm{~min}$ \\
V: $30 \mathrm{~cm}$ & $\sim 10 \mathrm{~min}$ & $\sim 25 \mathrm{~min}$ \\
V: $15 \mathrm{~cm}$ & $\sim 20 \mathrm{~min}$ & $\sim 50 \mathrm{~min}$ \\
\hline
\end{tabular}


WARTK user only needs to fix wide-lane DD ambiguities (that do not depend on the ionospheric activity) and these can be determined in a reliable way. This wide-lane ambiguity fixing, combined with the broadcast ionospheric corrections and associated sigmas computed by the CPF, provides the key for quick precise positioning with integrity. On the other hand, as indicated in the previous section, once the filter has converged the solution is mainly driven by the ionosphere-free combination of carrier measurements, which is not directly affected by the ionosphere.

[39] A second point to consider is the fact that the previous WARTK convergence time of 5-10 minutes found with real GPS data will be dramatically reduced with the threefrequency signals (i.e., Galileo). Indeed, as in the work by Hernández-Pajares et al. [2003], with three-frequency signals the wide-lane ambiguities can be fixed in a single epoch with the help of the accurate WARTK ionospheric corrections. Thence, the aforementioned level of accuracy will be achievable from the beginning (or, at least, after 30 seconds to allow the tropospheric estimate to converge).

[40] Note that, on the other hand, the given convergence time for the two-frequency signals corresponds to a cold start (mainly required to obtain a good value of the widelane ambiguity). In a warm start, high-precision positioning service (HPPS) would be resumed instantaneously. On the other hand, due to the fact that the carrier phase is the main observable in this approach, and that this can be modeled with pre-fit residuals to centimeter accuracy for static receivers, any anomalies can be detected much earlier in WARTK than in other systems mostly based on pseudorange data (such as SBAS), providing a shorter time to alarm.

\subsection{Accuracy and Integrity}

[41] The overall results indicate daily accuracy of 20 and $30 \mathrm{~cm}$ at the 95th percentile in the horizontal and vertical error components, respectively. This error is also protected by safety integrity levels, having daily 95 th percentiles of 1 meter and 2 meters for the horizontal and vertical protection levels, respectively. Notice that such figures are about one order of magnitude smaller than the performance obtained from EGNOS [see Ventura-Traveset and Flament, 2009].

[42] The vertical and horizontal protection levels (VPL and HPL) are given by $V P L=5.33 \cdot \beta \cdot \sigma_{V}$ and $H P L=6.18 \cdot \beta \cdot \sigma_{H}$, respectively. The values 5.33 and 6.18 are the K-factors associated with probabilities of misleading information (MI) of $10^{-7}$ and $510^{-9}$, respectively (see the derivation by Radio Technical Commission for Aeronautics [2001, Appendix J]). The sigmas, $\sigma_{V}$ and $\sigma_{H}$, are the corresponding vertical and horizontal standard deviations estimated in the user navigation filter, and $\beta$ is an inflating factor to guarantee overbounding of the actual errors by a Gaussian distribution. A value of $\beta=4.5$ has been adopted in this study, while $\sigma_{H}$ and $\sigma_{V}$ are extracted from the faultfree covariance matrix obtained directly from the navigation filter.

[43] The aforementioned value of $\beta$ can be obtained roughly as follows: once the filter has converged, the user position is driven by the second of the equations (5), so $\sigma_{H}$ and $\sigma_{V}$ mostly depend on the covariance element of $D D L c$ in equation (5) (i.e., $\sigma_{L c}$ ). In this paper, we have assumed $\sigma_{L c}=1 \mathrm{~cm}$, which is a relatively small value and does not take into account any modeling errors or potential biases introduced by the corrections. Moreover, one has to take into account that the observables in the second of the equations (5) are the DDs of $L c$ and that the non-differenced $L c$ is three times noisier than the $L 1$. Therefore, essentially, beta is a inflating factor for this non-conservative $\sigma_{L c}$ value. Note that one would obtain similar results, without needing the inflating factor, assuming a more conservative value of $\sigma_{L c}$ of $4.5 \mathrm{~cm}$ (instead of $1 \mathrm{~cm}$ ).

[44] In order to illustrate the navigation performance as a function of time for a given day and roving receiver, Figure 6 shows two typical examples of positioning performance, with the small positioning error bounded by safety integrity margins. These examples correspond to receiver ebre on 16 December (DOY 350) 2006, and lliv on 10 May (DOY 130) 2002.

[45] To illustrate the integrity performance achieved during the three periods analyzed, a helpful tool taken from a different field (civil aviation) is used, namely, the Stanford plot [see, e.g., Tossaint et al., 2007]. It consists of a histogram of positioning solutions in terms of actual error and protection level.

[46] The Stanford plots are shown in Figure 7 for the different data collection campaigns, merging all navigation solutions for all the rovers (bell, creu, ebre, and lliv) in the same plot in order to show the total user domain integrity.

[47] As shown, the position error is always bounded by the protection levels, that is, no MI event is found in any error component. Only near MIs (NMIs), i.e., $0.75<X P E /$ $X P L<1$, appear in the 2002 data campaign solutions for a single station and on a single day. These NMIs were experienced in the station creu on 3 May (DOY 123) 2002, and are due to an ionospheric interpolation problem that produced a drift in the ionospheric correction. On all the other days, the integrity margins are safe in all cases.

[48] Notably, at the 95th percentile, thresholds of $H P E<6.9 m$ and $V P E<2.9 m$ for positioning accuracy and $H P L<17 \mathrm{~m}$ and $V P L<5.3 \mathrm{~m}$ for protection levels, in line with the CAT-III requirements for accuracy and protection levels for civil aviation (values which are still under discussion [see, e.g., Xie, 2004]), are fulfilled in the representative periods studied. Although more studies must be carried out, these results, taking into account that the L2 has been used rather than the incoming L5 signal, suggest a potential way to achieve CAT-III requirements at a continental scale using the WARTK technique.

\subsection{Extrapolation of Results to a Multi-constellation System}

[49] The aforementioned results are based on real twofrequency measurements and a single constellation (i.e., the current GPS signal). Nevertheless, an extrapolation of performances to a multi-constellation system involving dualfrequency (GPS) and three-frequency (Galileo) measurements can be made from the results of the study performed using simulated GPS and Galileo data.

[50] The simulation was performed at the ESA European Space Research and Technology Centre involving a Spirent signal simulator and two GNSS receivers: a Septentrio PolaRx 2 for GPS L1 and L2 frequencies and an IfEN NavXRPS for Galileo signals (E1, E5a and E5b). The GNSS signals for seven permanent receivers and one user receiver 
ebre: VPE: mean $=0.03 \mathrm{~m}, 95 \mathrm{th}=0.09 \mathrm{~m} ; \mathrm{VPL}:$ mean $=0.91 \mathrm{~m}, 95 \mathrm{th}=1.33 \mathrm{~m}$

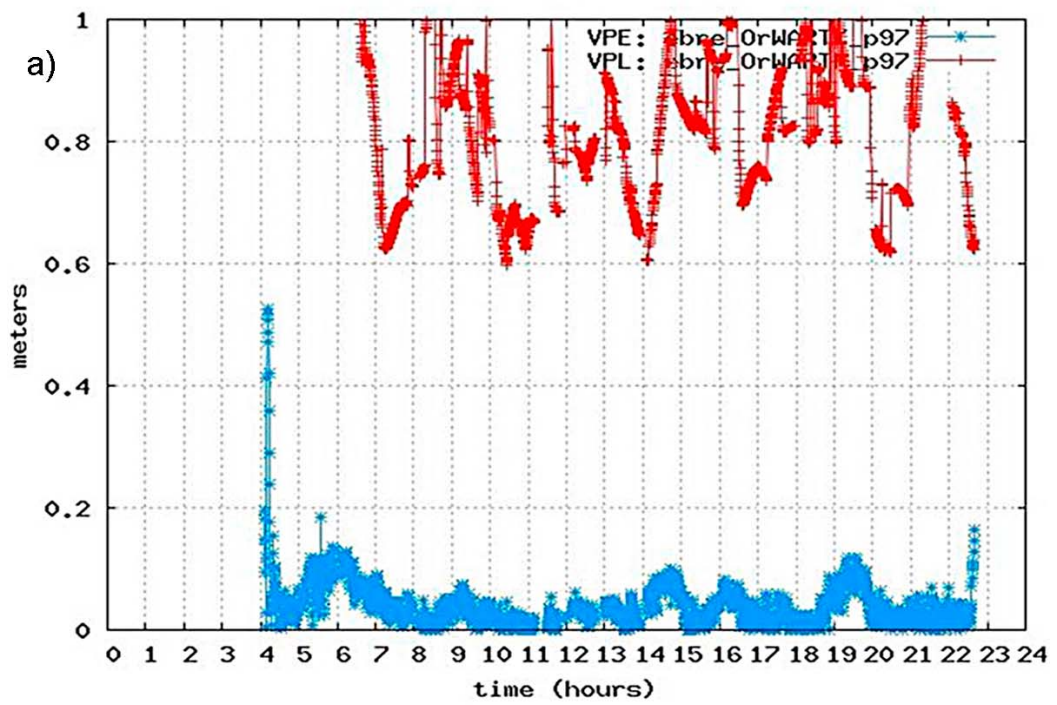

Iliv : VPE: mean $=0.03 \mathrm{~m}, 95 \mathrm{th}=0.08 \mathrm{~m}$; VPL: mean $=0.96 \mathrm{~m}, 95 \mathrm{th}=1.28 \mathrm{~m}$

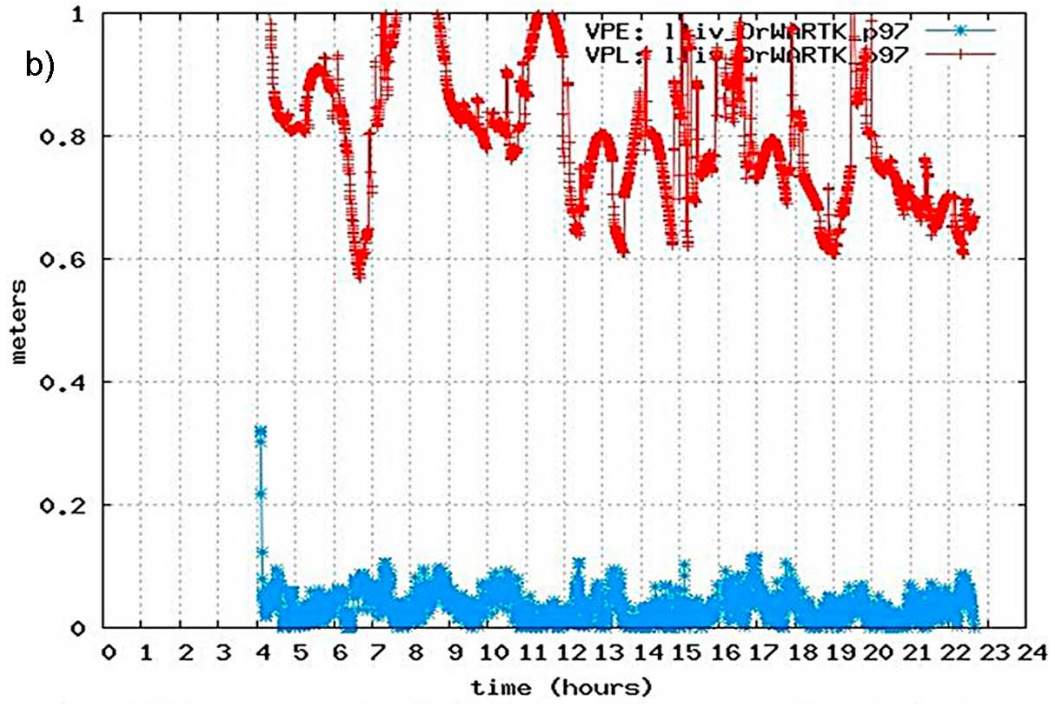

ebre : HPE: mean $=0.02 \mathrm{~m}, 95 \mathrm{th}=0.06 \mathrm{~m} ; \mathrm{HPL}:$ mean $=0.49 \mathrm{~m}, 95 \mathrm{th}=0.74 \mathrm{~m}$

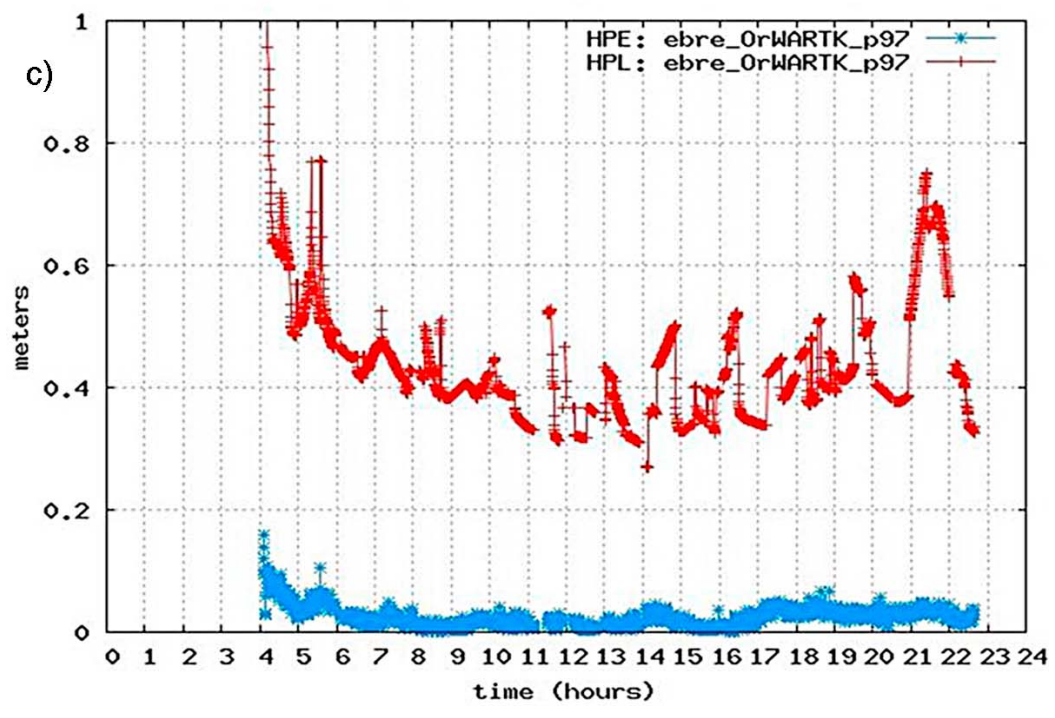

Figure 6

10 of 14 
Iliv: HPE: mean $=0.02 \mathrm{~m}, 95 \mathrm{th}=0.04 \mathrm{~m}$; HPL: mean $=0.49 \mathrm{~m}, 95 \mathrm{th}=0.68 \mathrm{~m}$
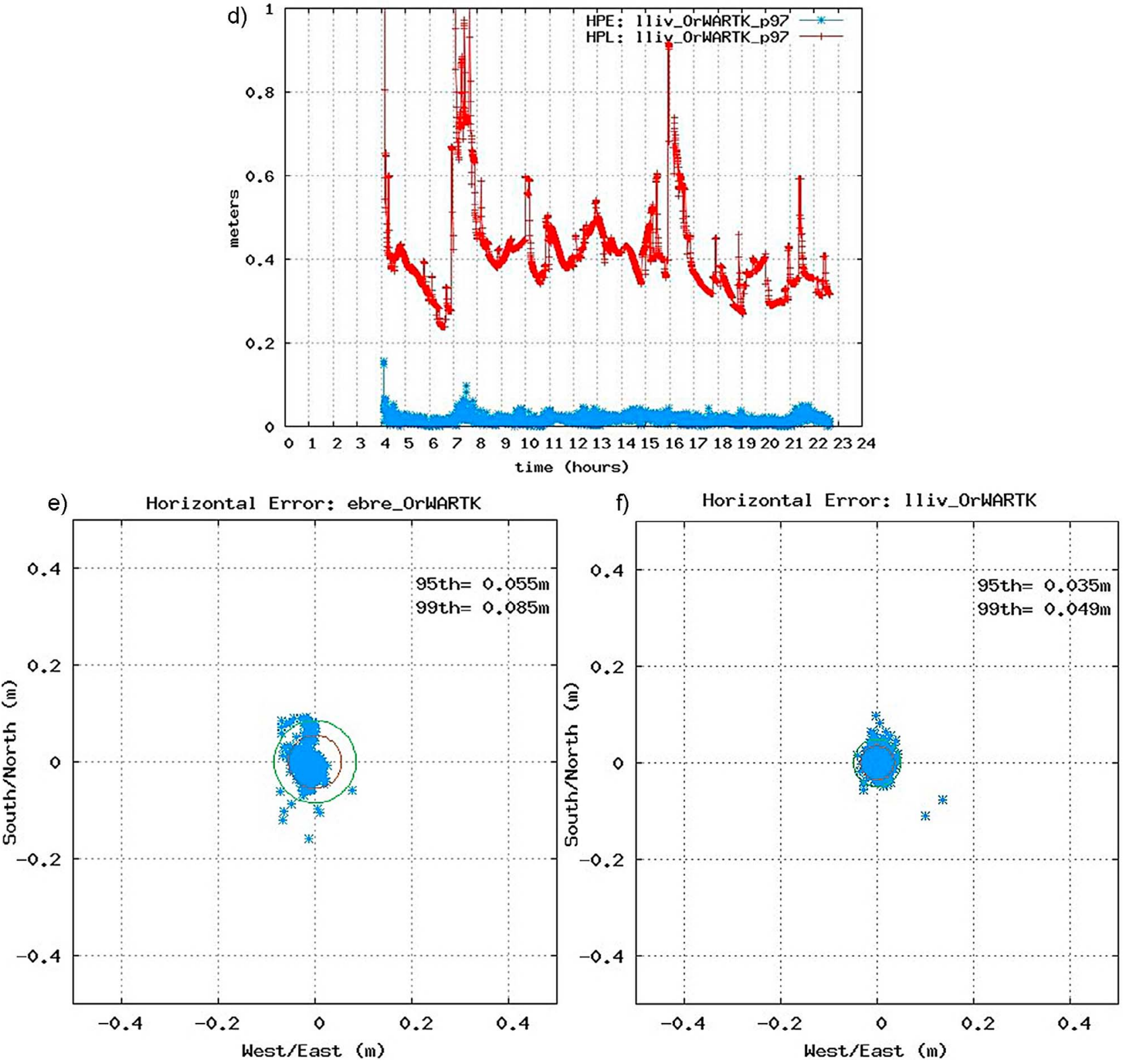

Figure 6. (continued)

were simulated by entering the coordinates of the receivers into the signal simulator. The simulated locations of the seven permanent receivers corresponded to that of six actual EGNOS RIMS (A001, A003, A010, A015, A021 and A034) plus an additional IGS receiver (STAS in the south of Norway). The user receiver was placed in Delft (Holland) at $413 \mathrm{~km}$ from the nearest reference receiver (A015).

[51] The signal simulator has the capability of simulating realistic effects on the GNSS signals such as ionospheric and tropospheric delays, multipath phenomena, etc., but, in order to have more control over the delays, none of these effects was added in the simulation process. Accordingly, the pseudorange thermal noise in the data acquisition process was the most significant effect in the GNSS signals gathered directly from the receivers (with standard deviations from $10 \mathrm{~cm}$ to $20 \mathrm{~cm}$, depending on the frequencies). Ionospheric delays were simulated using the International Reference Ionosphere model [Bilitza, 2001] assuming moderate ionospheric conditions, while tropospheric delays were simulated using a different mapping function from the one used in the

Figure 6. Two examples of user domain performance in actual GPS data scenarios: for (a, c, e) ebre (2006 DOY 350) and (b, d, f) lliv (2002 DOY 130) rovers with baselines of $315 \mathrm{~km}$ and $127 \mathrm{~km}$, respectively. Figures $6 \mathrm{a}$ and $6 \mathrm{~b}$ show the vertical position error (blue) and protection levels (red), and Figures $6 \mathrm{c}$ and $6 \mathrm{~d}$ show the horizontal position error (blue) and protection levels (red). The mean values and 95th percentiles for position error XPE and protection levels XPL are shown at top of each plot. Figures 6e and 6f show the North-south and East-west position error and the 95th and 99th percentiles. 
2002no_reset [OrgW_p97, 7200_300s] N=166360

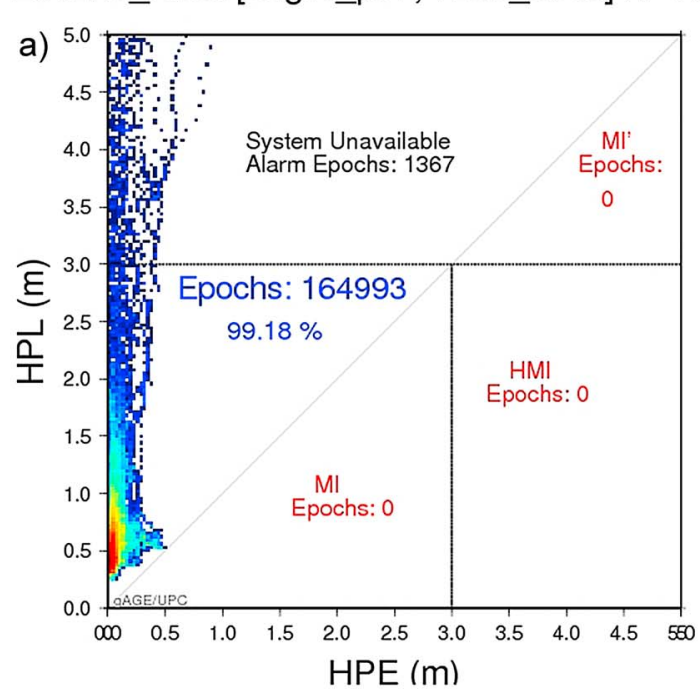

2004no_reset [OrgW_p97, 7200_300s] N=201809

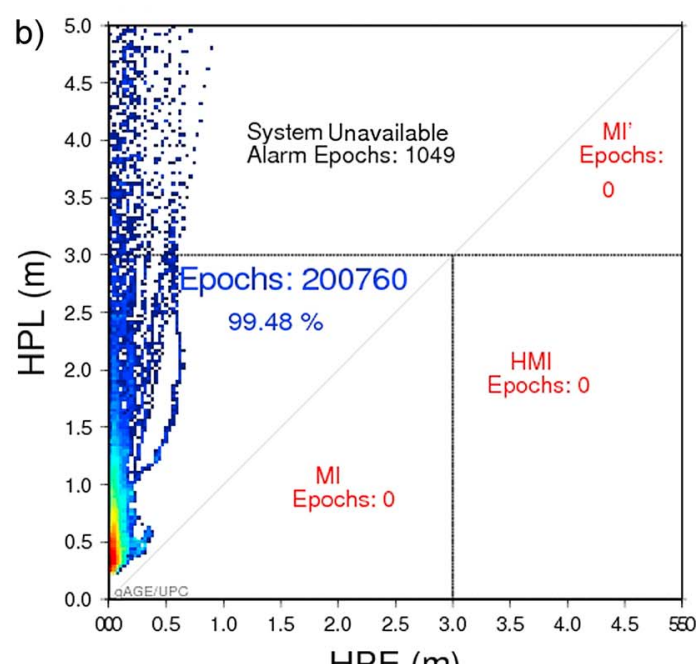

HPE (m)

2006no_reset [OrgW_p97, 7200_300s] N=219906

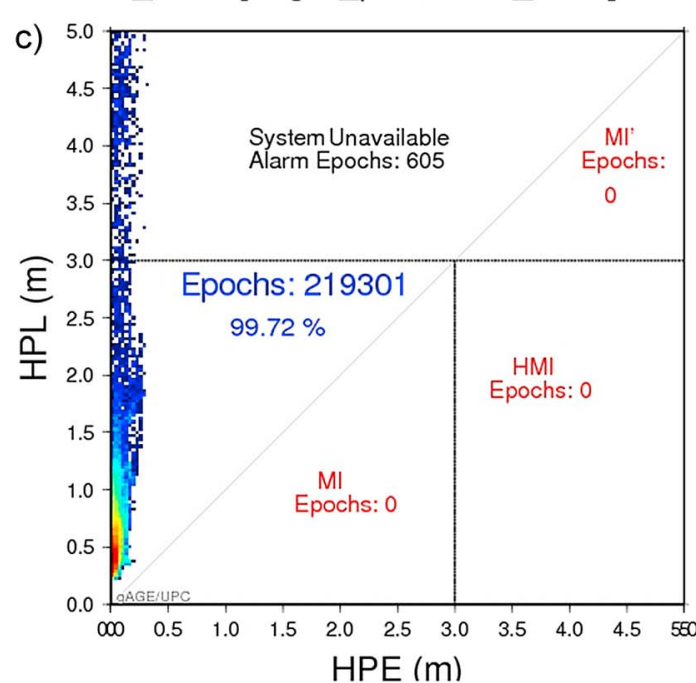

2002no_reset [OrgW_p97, 7200_300s] N=166360

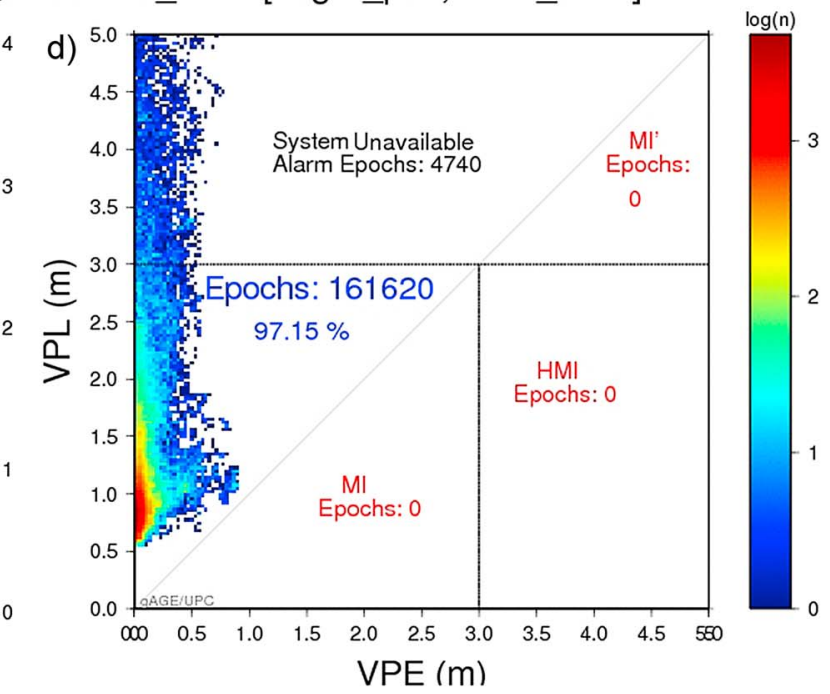

2004no_reset [OrgW_p97, 7200_300s] N=201809

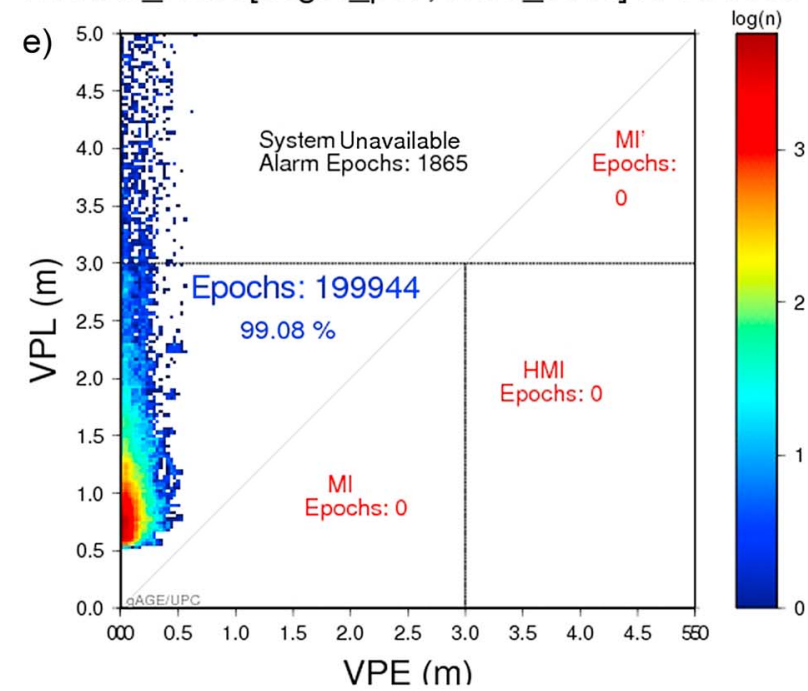

2006no_reset [OrgW_p97, 7200_300s] N=219906
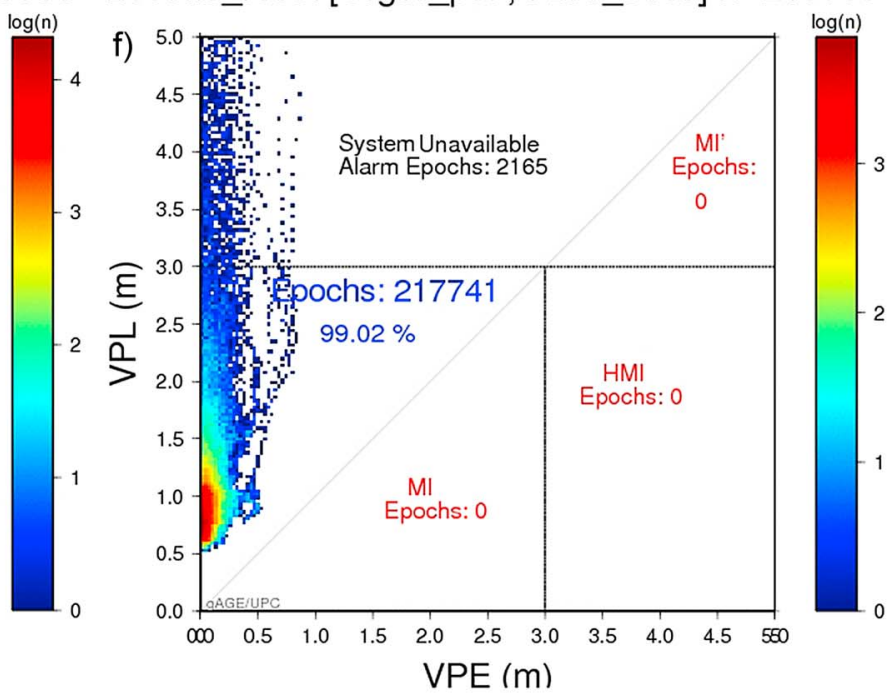

Figure 7. $(\mathrm{a}-\mathrm{c})$ Horizontal and $(\mathrm{d}-\mathrm{f})$ vertical Stanford plots for different data collection campaigns with actual GPS data scenarios: 2002 (Figures 7a and 7d), 2004 (Figures 7b and 7e), and 2006 (Figures 7c and $7 \mathrm{f}$ ). All navigation solutions for all stations in each data set are merged into the same plot. 

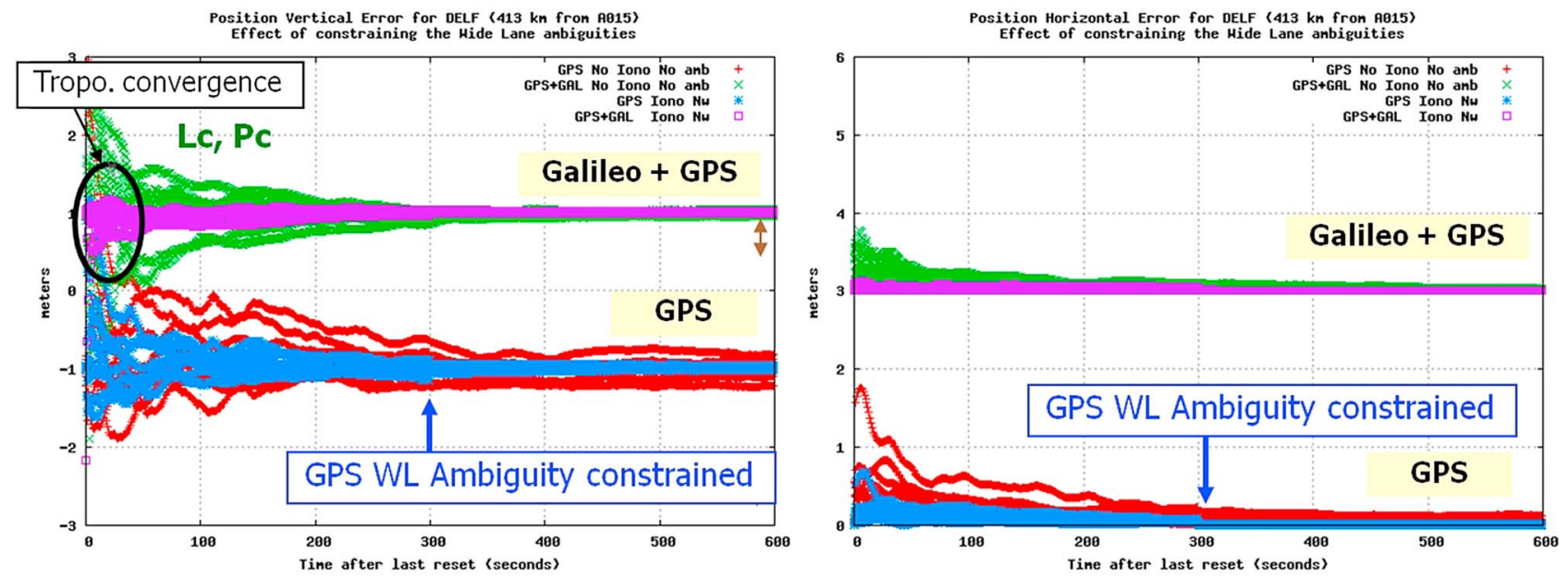

Figure 8. Generated using signal simulated data (Delf station navigated relative to A015, baseline $413 \mathrm{~km}$ ). The plot shows a comparison of the convergence of the positioning error with the WARTK (blue and magenta) and the standard differential positioning (red and green)techniques. Each plot shows the results using single constellation (GPS) and multi-constellation (GPS + Galileo). The (left) vertical and (right) horizontal components are shown. The receiver was reset every 5 minutes. All the solutions are merged into the same 600-second window. Note a jump in the accuracy when constraining the wide-lane ambiguity (see explanation in section 4). The horizontal axis is the time since receiver reset. The vertical axis shows the positioning error, conveniently shifted to improve visibility.

filter equations (1) and (5). On the other hand, pseudorange multipath was not added to the signals, and thence, the results found in this section for each technique separately can be considered as optimistic, especially for the standard differential positioning in which the convergence time depends on the pseudorange noise. Nevertheless, the relative performance of WARTK and standard differential positioning (which is the main focus of this study) shows realistic figures and, thence, they can be used as a baseline to extrapolate the results obtained with the currently considered GPS signal (single constellation), to a multi-constellation with GPS + Galileo signals.

[52] The results for this multi-constellation simulated scenario are reported in Figure 8 and Table 3. They can be summarized as follows:

[53] 1. The results from the simulated GPS data (i.e., simulated dual-frequency data from a single constellation) show a reduction by a factor of 8 and 3, respectively, for horizontal and vertical error when ionospheric corrections are used. This is similar to the improvement obtained with real GPS data (Table 2).

[54] 2. In a multi-constellation scenario with three-frequency signals, the convergence time is reduced to few seconds, thanks to the use of the extra-wide-lane combination [see also Hernández-Pajares et al., 2003].

[55] 3. A factor of two improvement in the daily 95th percentiles of accuracy (XPE) and protection levels (XPL) is found for a multi-constellation with respect to the single constellation.

\section{Conclusions}

[56] The Wide Area Real Time Kinematic technique relies on the broadcasting of accurate ionospheric corrections computed in real-time to allow the users to achieve high accuracy positioning quickly and on a continental scale. Considerable previous research has been carried out to assess its feasibility. In this study, a more detailed characterization was performed, showing results over larger and more representative data sets, including analysis of position integrity performance.

[57] An EGNOS-like reference station network was built from the public domain IGS permanent GPS receiver network. The dual frequency measurements from these stations were processed by the WARTK CPF, which combines a geodetic and an accurate ionospheric model to compute the WARTK corrections. The carrier phase ambiguities, double differenced between stations and satellites, were fixed and used in the CPF filter as constraints in order to give more strength to the parameters estimation. In this way, highly accurate ionospheric corrections, which are the key factor in the WARTK navigation technique, were calculated among the satellite clocks, DCBs and other products.

Table 3. WARTK Expected Performance ${ }^{a}$

\begin{tabular}{|c|c|c|}
\hline \multirow{2}{*}{$\begin{array}{c}\text { Convergence Time } \\
\text { (to } 20 \mathrm{~cm} \text { Hor. } \\
\text { and } 30 \mathrm{~cm} \text { Vert.) } \\
\text { [Improvement Factor] }\end{array}$} & \multicolumn{2}{|c|}{ Daily 95th Percentile } \\
\hline & Accuracy (XPE) & Integrity (XPL) \\
\hline \multicolumn{3}{|c|}{ Single Constellation 2-Frequency Signals } \\
\hline Hor. $<5 \min [8 \times]$ & $<20 \mathrm{~cm}$ & $\simeq 100 \mathrm{~cm}$ \\
\hline Vert. $<10 \min [3 \times]$ & $<30 \mathrm{~cm}$ & $\simeq 200 \mathrm{~cm}$ \\
\hline \multicolumn{3}{|c|}{ Multi-Constellation 2 and 3-Frequency Signals } \\
\hline Hor. A few seconds & $<10 \mathrm{~cm}$ & $\simeq 50 \mathrm{~cm}$ \\
\hline Vert. A few seconds & $<15 \mathrm{~cm}$ & $\simeq 100 \mathrm{~cm}$ \\
\hline
\end{tabular}

${ }^{\mathrm{a}}$ This assessment considered the same $\mathrm{K}$-factors used in civil aviation to calculated the protection levels (i.e., associated with 5E-9 and 1E-7 probabilities, for the vertical and horizontal protection levels respectively). 
[58] The user domain performance assessment was carried out using four IGS permanent dual frequency GPS receivers, that were differentially navigated relative to a reference station with baselines ranging from $117 \mathrm{~km}$ to $315 \mathrm{~km}$. Although such receivers are IGS fixed sites, they have been treated as rovers, that is, using pure kinematic mode and emulating real-time conditions. As the ionosphere is a key factor for the WARTK positioning approach, the data sets used in this assessment consist of three data collection campaigns of four weeks each, covering different ionospheric conditions. The first data collection campaign was in 2002, under solar maximum conditions, and includes three disturbed days; the second in 2004 with mid solar cycle conditions; and the third in 2006, with low solar activity, but still including a disturbed day.

[59] The results show that high accuracy navigation is achieved quickly with the WARTK approach thanks to the use of precise ionospheric corrections and the wide-lane ambiguity constraints. Daily 95th percentiles of 20 and 30 centimeters are achieved in the horizontal and vertical error components, respectively. On average, these accuracies are reached within 5 and 10 minutes, respectively. This quick high accuracy is protected by safe integrity margins, with protection levels having daily 95th percentiles typically less than 1 meter in horizontal and 2 meters in vertical components. These figures show the feasibility of fulfilling CAT-III requirements for accuracy and protection levels.

[60] The aforementioned figures are expected to improve with three-frequency (e.g., Galileo) signals, thanks to the single epoch fixing of wide-lane ambiguities, these accuracy levels being reached within a few seconds. Moreover, in a multi-constellation scenario, the 95th percentiles of accuracy and protection levels percentiles can be expected to improve by about a factor of 2 due to instantaneous ambiguity fixing, as well as the better satellite geometry. Finally, the straightforward fixation of the extra-wide-lane carrier phase ambiguity means that the integrity will also improve with the new signals.

[61] As a summary, Table 3 lists the expected performances of the WARTK approach based on the results obtained in this assessment with real two-frequency GPS signals. The values for the multi-constellation are extrapolated from these figures using the results based on two-frequency GPS and three-frequency Galileo simulated signals.

[62] Acknowledgments. Most of this work was carried out in the context of the Multi-constellation Regional System (MRS) project, within the ESA GNSS Evolution Programme. In addition, it was partially supported by the Spanish Ministry of Science and Innovation under the CTM2010-21312-C03-02 and ESP2007-62676 projects.

\section{References}

Bilitza, D. (2001), International Reference Ionosphere 2000, Radio Sci., 36(2), 261-275.

Colombo, O. L., M. Hernández-Pajares, J. M. Juan, J. Sanz, and J. Talaya (1999), Resolving carrier-phase ambiguities on the fly, at more than
$100 \mathrm{~km}$ from nearest reference site, with the help of ionospheric tomography, paper presented at ION GPS-99, Inst. of Navig., Nashville, Tenn. Datta-Barua, S., T. Walter, E. Altshuler, J. Blanch, and P. Enge (2005), Dst as an indicator of potential threats to WAAS integrity and availability, paper presented at the 18th International Technical Meeting, Satell. Div., Inst. of Navig., Long Beach, Calif.

Grejner-Brzezinska, D. A., P. Wielgosz, I. Kashani, D. A. Smith, D. S. Robertson, G. L. Mader, and A. Komjathy (2006), The impact of severe ionospheric conditions on the accuracy of kinematic position estimation: Performance analysis of various ionosphere modelling techniques, Navigation, 53(3), 203-217.

Hernández-Pajares, M., J. M. Juan, and J. Sanz (1997), Neural network modelling of the ionospheric electron content at global scale using GPS data, Radio Sci., 32(3), 1081-1089, doi:10.1029/97RS00431.

Hernández-Pajares, M., J. M. Juan, J. Sanz, and O. C. Colombo (1999), Ionospheric determination and its application to real-time GPS ambiguity resolution, paper presented at ION GPS-99, Inst. of Navig., Nashville, Tenn.

Hernández-Pajares, M., J. M. Juan, J. Sanz, and O. L. Colombo (2002), Improving the real-time ionospheric determination from GPS sites at very long distances over the equator, J. Geophys. Res., 107(A10), 1296, doi:10.1029/2001JA009203.

Hernández-Pajares, M., J. M. Juan, J. Sanz, and O. C. Colombo (2003), Feasibility of wide-area subdecimeter navigation with GALILEO and modernized GPS, IEEE Trans. Geosci. Remote Sens., 41(9), 2128-2131.

Hernández-Pajares, M., J. M. Juan, J. Sanz, R. Orús, A. García-Rodríguez, and O. C. Colombo (2004), Wide area real time kinematics with Galileo and GPS signals, paper presented at ION GNSS-2004, Inst. of Navig., Long Beach, Calif.

Hernández-Pajares, M., J. M. Juan, and J. Sanz (2006), Medium-scale traveling ionospheric disturbances affecting GPS measurements: Spatial and temporal analysis, J. Geophys. Res., 111, A07S11, doi:10.1029/ 2005JA011474.

Hernández-Pajares, M., et al. (2008), Feasibility study of a European wide area real time kinematic system, paper presented at 4th ESA Workshop on Satellite Navigation User Equipment Technologies, Eur. Space Res. and Technol. Cent., Noordwijk, Netherlands.

Hernández-Pajares, M., et al. (2010), Wide-Area RTK, Inside GNSS, 5(2), $35-46$.

Juan, J. M., A. Rius, M. Hernández-Pajares, and J. Sanz (1997), A twolayer model of the ionosphere using Global Positioning System data, Geophys. Res. Lett., 24, 393-396, doi:10.1029/97GL00092.

Misra, P., and P. Enge (2010), Global Positioning System: Signals, Measurements, and Performance, 2nd ed., Gamma-Jamuna, Lincoln, Mass.

Orús, R., L. R. Cander, and M. Hernández-Pajares (2007), Testing regional vTEC maps over Europe during the 17-21 January 2005 sudden space weather event, Radio Sci., 42, RS3004, doi:10.1029/2006RS003515.

Radio Technical Commission for Aeronautics (2001), Minimum operational performance standards for Global Positioning System/Wide Area Augmentation System airborne equipment, Rep. DO-229C, Washington, D. C.

Tossaint, M., J. Samson, F. Toran, J. Ventura-Traveset, M. Hernández-Pajares, J. M. Juan, J. Sanz, and P. Ramos-Bosch (2007), The Stanford-ESA integrity diagram: A new tool for the user domain SBAS integrity assessment, Navigation, 54(2), 153-162.

Ventura-Traveset, J., and D. Flament (Eds.) (2009), EGNOS: The European Geostationary Navigation Overlay System: A Cornerstone of Galileo, Eur. Space Agency Spec. Publ., ESA SP-1303.

Wanninger, L. (2004), Ionospheric disturbance indices for RTK and network RTK positioning, paper presented at ION GPS/2004, Inst. of Navig., Long Beach, Calif.

Xie, G. (2004), Optimal on-airport monitoring of the integrity of GPS-based landing systems, $\mathrm{PhD}$ dissertation, Stanford Univ., Stanford, Calif.

A. Aragón-Àngel, M. Hernández-Pajares, J. M. Juan, D. Salazar, and J. Sanz, Research Group of Astronomy and Geomatics, Technical University of Catalonia, E-08034 Barcelona, Spain. (jaume@ma4.upc.edu)

J. Samson and M. Tossaint, European Space Research and Technology Centre, European Space Agency, NL-2201 AZ Noordwijk, Netherlands. 